\title{
A New Approach for RRR Determination of Niobium Single Crystal Based on AC Magnetic Susceptibility
}

\author{
A. Ermakov, A. V. Korolev*, W. Singer, X. Singer
} presented by A. Ermakov

Deutsches Elektronen-Synchrotron, Hamburg, Germany

* Institute of Metal Physics, Ekaterinburg, Russia 


\section{OUTLINE}

- Introduction

- Main principles of RRR determination

Single crystal samples

Equipment

RRR data obtained by AC magnetic susceptibility

Comparison with RRR obtained by DC method

Summary 


\section{INTRODUCTION}

Residual resistivity ratio (RRR) value is an important characteristic of material purity.

$A C$ magnetic susceptibility of a number of single crystal niobium samples for different orientations of type $\langle 100\rangle,\langle 011\rangle,\langle 111\rangle$ and treatments (BCP 70, $150 \mu \mathrm{m}$, annealing $800^{\circ} \mathrm{C} / 2 \mathrm{~h}$ ) were measured. The RRR value was determined on base of these results using a relation between the imaginary part $\chi$ " of $A C$ magnetic susceptibility at low frequency $f$ of $A C$ magnetic field and resistivity $\rho$ of the sample: $\chi "=k^{*} f l \rho$. 


\section{Main principles of RRR determination}

The $A C$ susceptibility caused by eddy current can be expressed for spherical sample in terms of it radius $\alpha$, and the skin penetration depth $\delta$ :

$$
\begin{aligned}
& \chi_{A C}=\chi^{\prime}+i \chi^{\prime \prime} \quad \chi^{\prime}=\frac{9}{4}\left(\frac{\delta}{a}\right) \frac{[\sinh (2 a / \delta)-\sin (2 a / \delta)]}{[\cosh (2 a / \delta)-\cos (2 a / \delta)]}-\frac{3}{2} \\
& \chi^{\prime \prime}=\frac{9}{4}\left(\frac{\delta}{a}\right) \frac{[\sinh (2 a / \delta)+\sin (2 a / \delta)]}{[\cosh (2 a / \delta)-\cos (2 a / \delta)]}-\frac{9}{4}\left(\frac{\delta}{a}\right)^{2} \\
& \delta=1 /\left(\pi \mu_{0} \mu \sigma f\right)^{0.5}=\left(\rho /\left(\pi \mu_{0} \mu f\right)^{0.5}\right.
\end{aligned}
$$

$\mu_{0}=4 \pi \times 10^{-7} \mathrm{H} / \mathrm{m} ; \mu$ - the relative permeability; $\rho$ - resistivity; $f$ - frequency. $A C$ method: at low $f-\chi$ " can be expressed as $\chi$ " $=A 1+A 2^{*} f$. In homogeneous sample $A 1=0, A 2=k^{*} \sigma$ ( $k=$ const); $\sigma=1 / \rho ; 1 / A 2=\rho / k ; \sigma-$ electrical conductivity

$$
\chi^{\prime \prime}=k^{*} \mathrm{f} / \rho
$$

$$
R R R=\rho_{300 K} / \rho_{4.2 K}
$$

- Magnetic susceptibility of superconductors and other spin systems, Ed. By Robert A. Hein et. al., Plenum Press New York, 1991, page. 213 [A. F. Khoder, M. Gouach, Early theories of X' and X" of superconductors for controversial aspects] 


\section{Single crystal samples}

- Sample N1 (as delivered)

- Sample N2, BCP, $70 \mu \mathrm{m}$

- Sample N3, BCP, $150 \mu \mathrm{m}$

\section{$800^{\circ} \mathrm{C} / 2 \mathrm{~h}$}

The single crystal samples of company Heraeus have

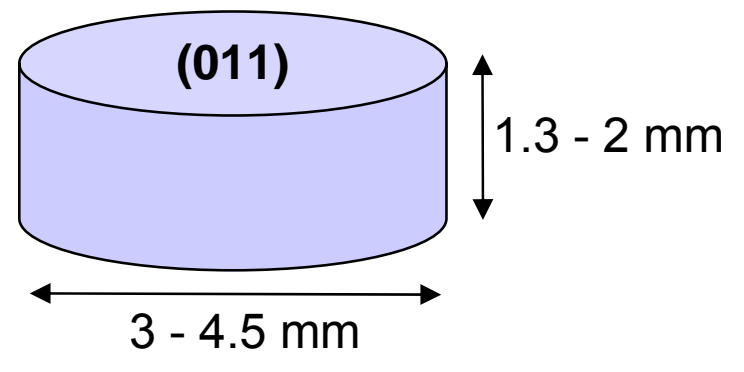
been used. The samples were cut out using EDM method.

Magnetic field applied along directions of type $\langle 100\rangle,\langle 011\rangle,\langle 111\rangle$ 
Sample N1 (as delivered) magnetic field along $<111>$

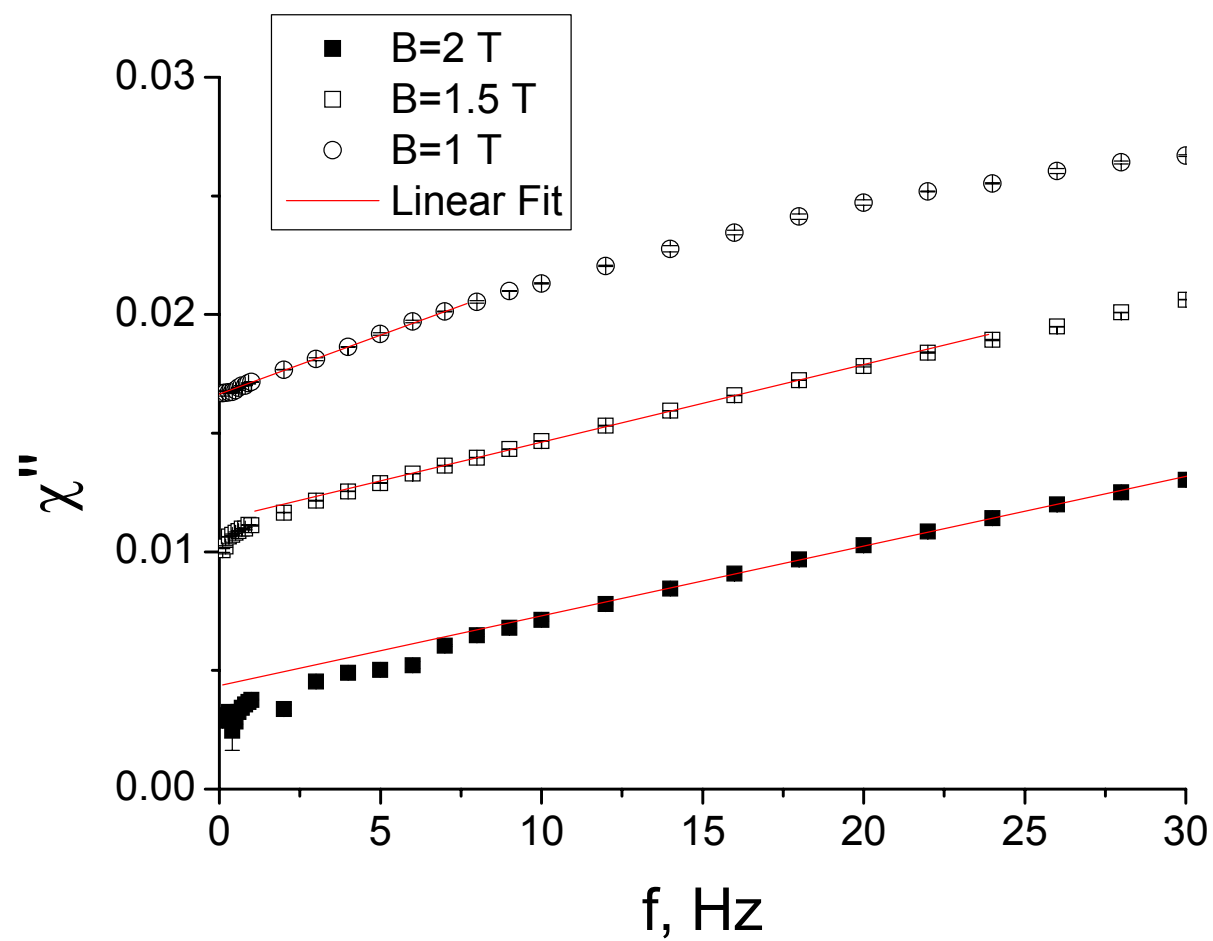

\section{frequency extrapolation}

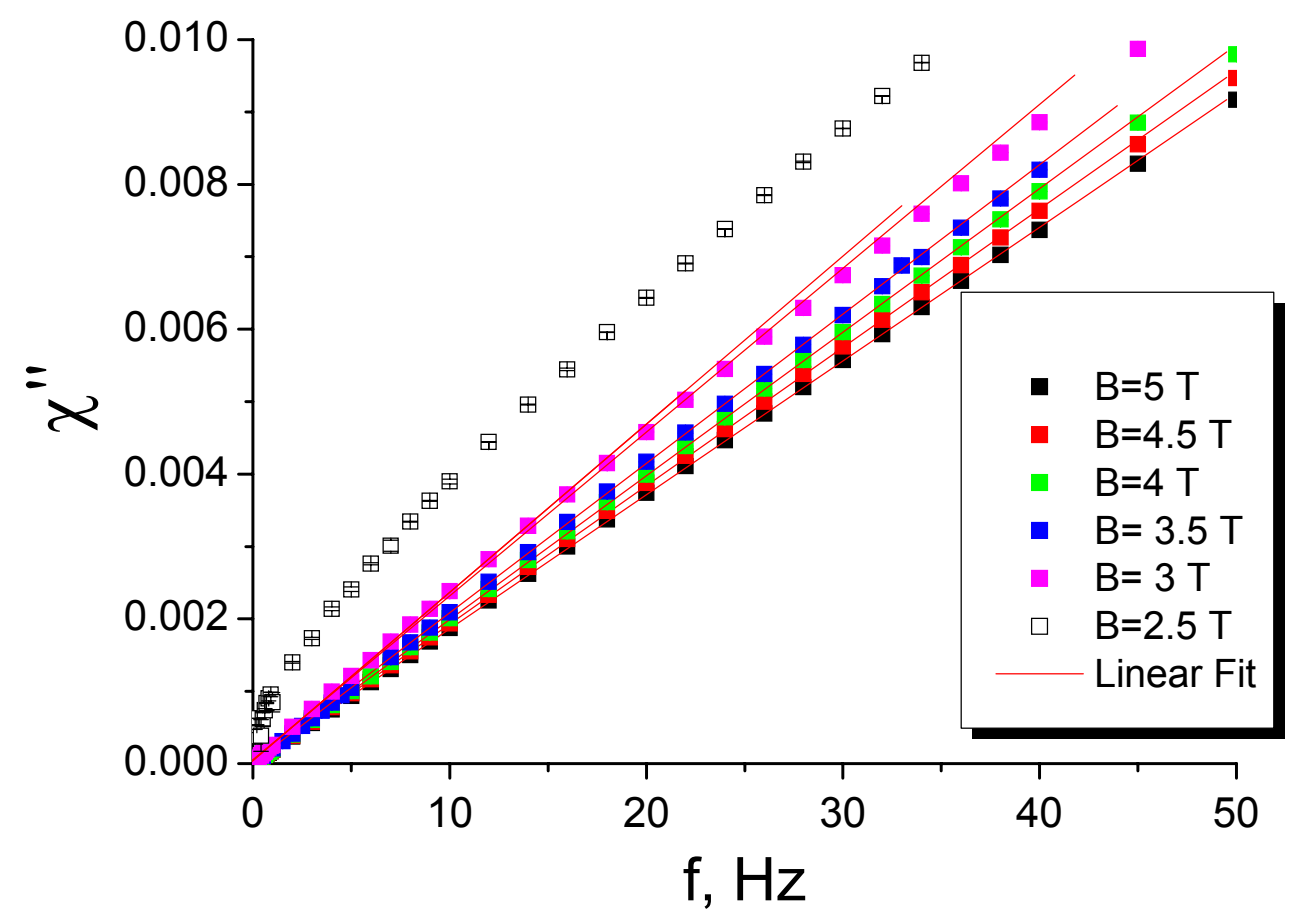

Frequency dependencies of imaginary part of AC-susceptibility for different values of applied magnetic field. At low frequency at $B<3 T$ observed the scattering of the points (left figure). At $B \geq 3 \mathrm{~T}$ change of the curve slope (right figure). 


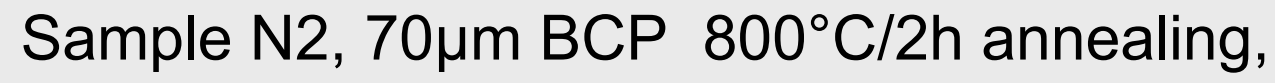
magnetic field along [100], $\langle 011\rangle,<111\rangle$
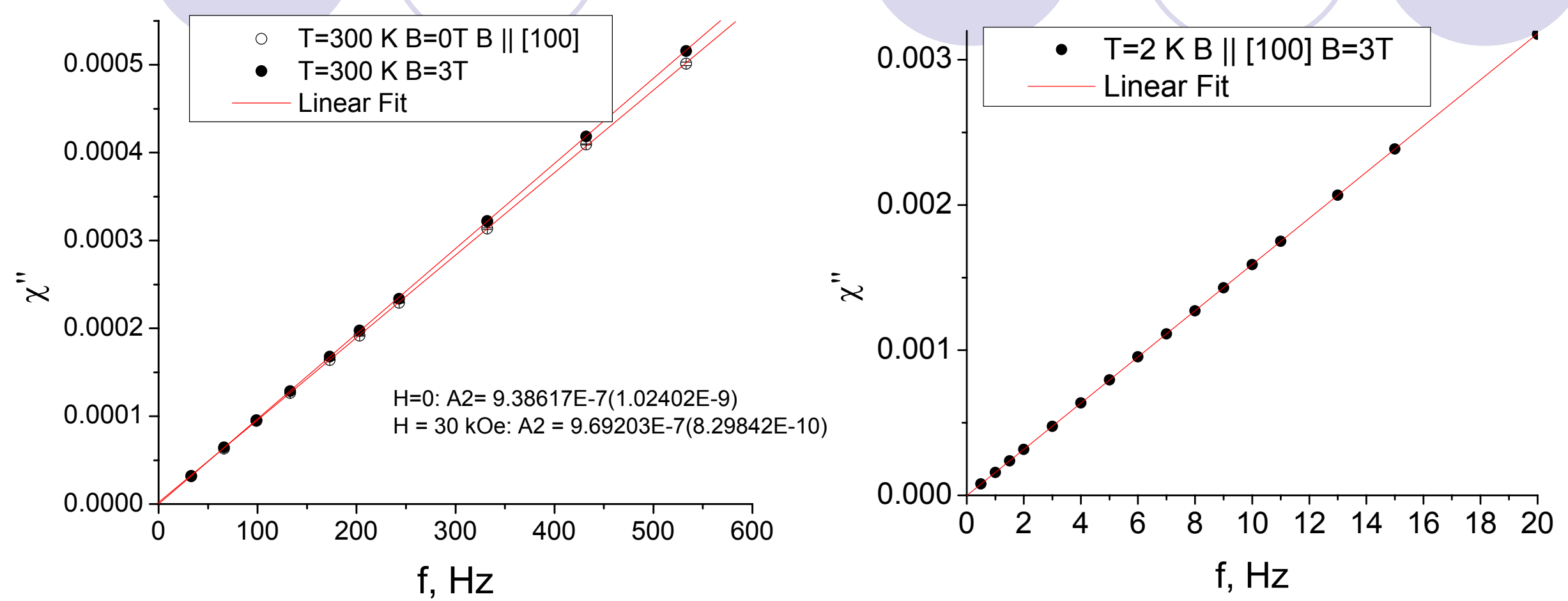

Frequency dependencies of imaginary part of susceptibility at $B=0 ; 3 T(T=2 K ; 300 K)$. Angle between the curves at $B=0 ; 3 T(T=300 K)$ shows the small magnetoresistivity.

\begin{tabular}{|c|c|}
\hline $\mathbf{B = 3} \mathrm{T} ; \mathbf{B} \boldsymbol{\|}$ & $\mathbf{R R R}$ \\
\hline$[100]$ & $169(\mathbf{2 0 7} \mathbf{B}=\mathbf{0 T})$ \\
\hline$<011>$ & 198 \\
\hline$<111>$ & 226 \\
\hline
\end{tabular}




\section{Sample $\mathrm{N} 2,70 \mu \mathrm{m} \mathrm{BCP} 800^{\circ} \mathrm{C} / 2 \mathrm{~h}$} annealing, magnetic field along [100]

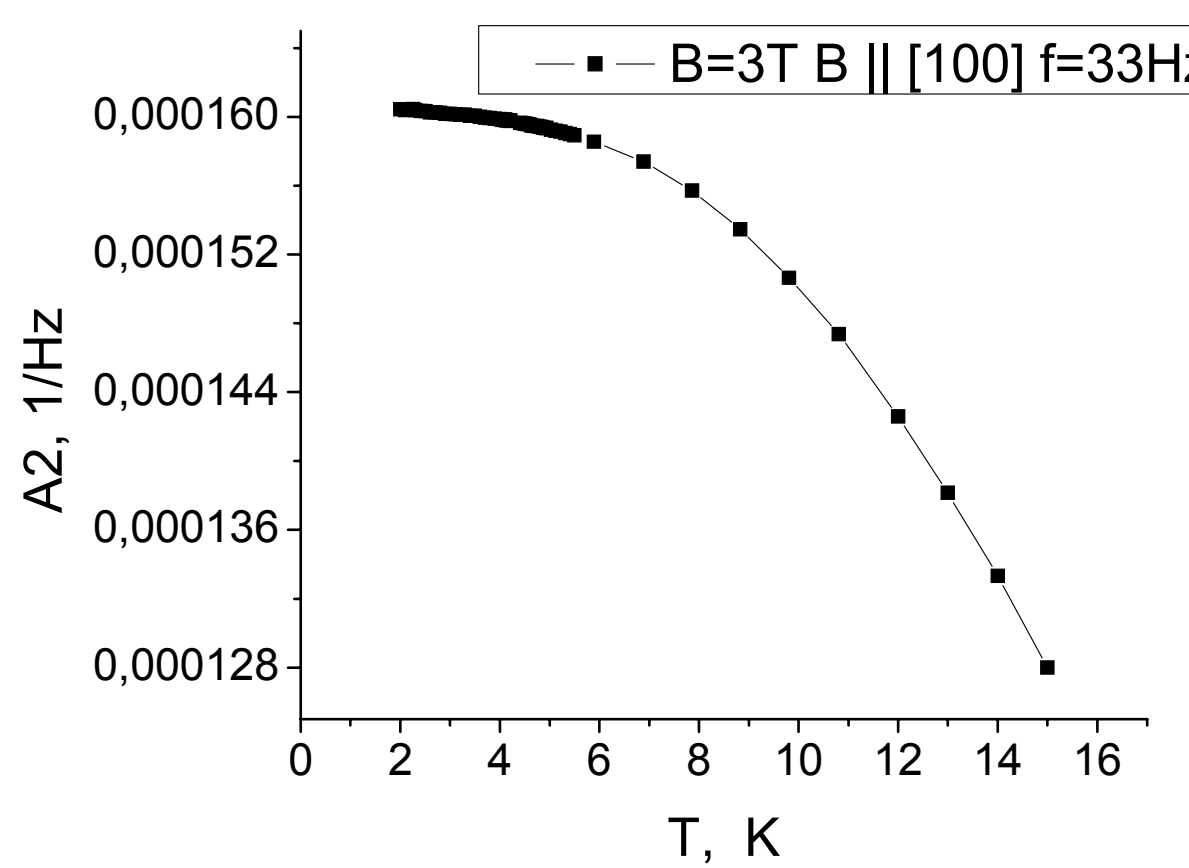

RRR=166 (B || [100]) by temperature extrapolation method RRR=169 (B || [100]) by frequency extrapolation method

\section{temperature extrapolation}

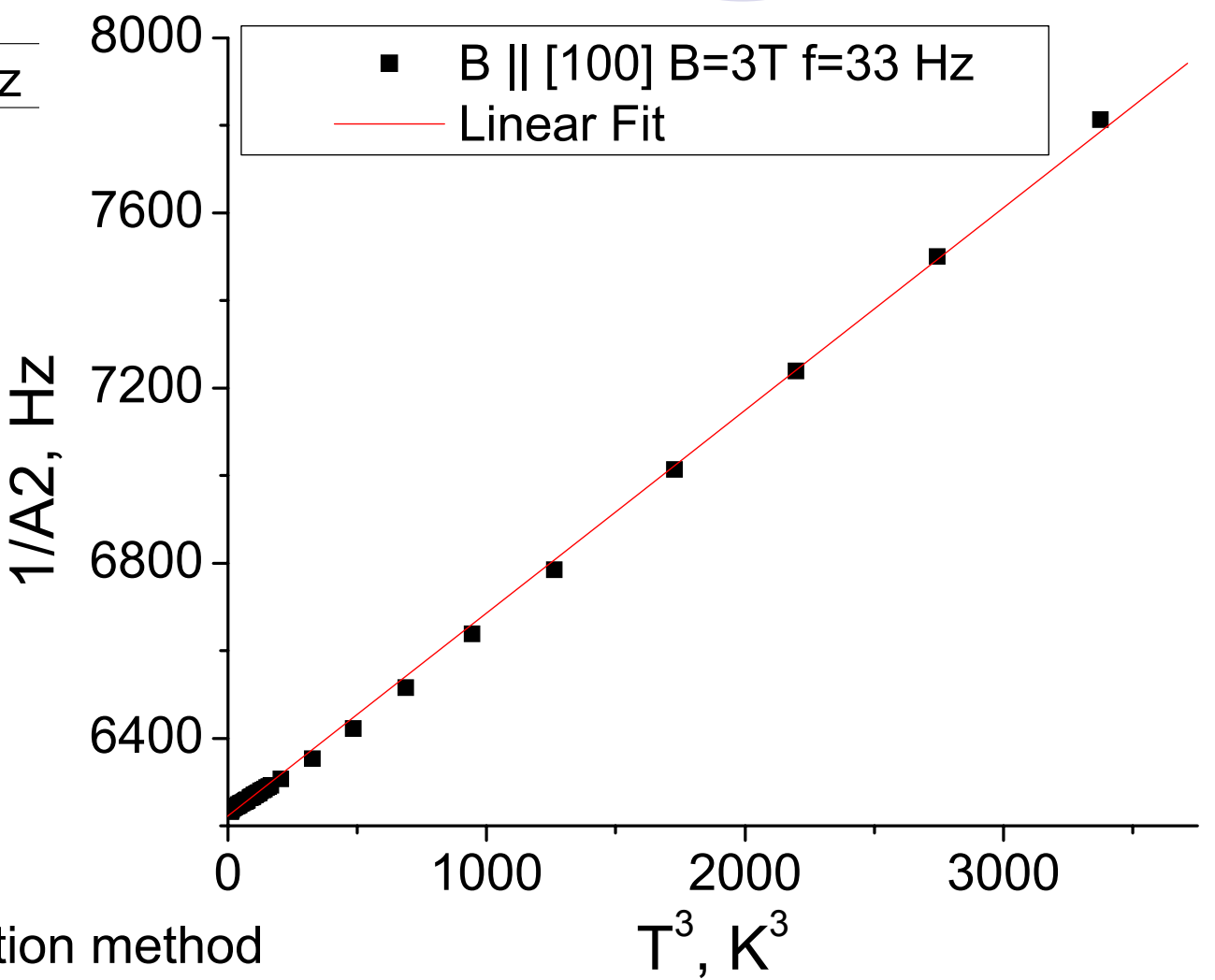

1/A2 dependence of $T^{3}$

correlation of RRR values obtained by frequency and temperature extrapolation 
Sample N3, $150 \mu \mathrm{m}$ BCP $800^{\circ} \mathrm{C} / 2 \mathrm{~h}$ annealing magnetic field along [100]

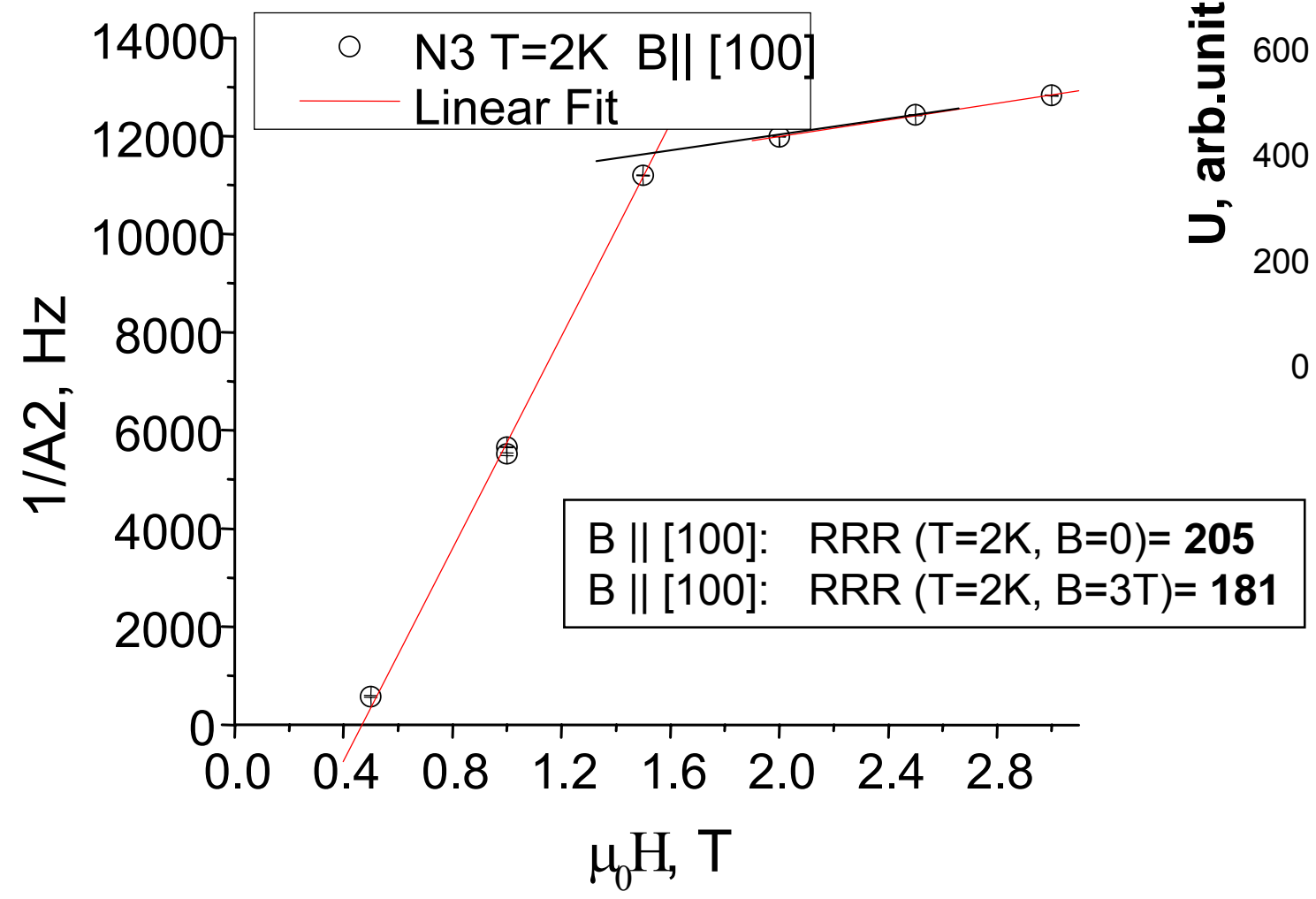

At $B \geq 1.5$ T curve $1 / A 2$ vs $B$ follows the Kapitza law: $R=K^{\star} f(B)$

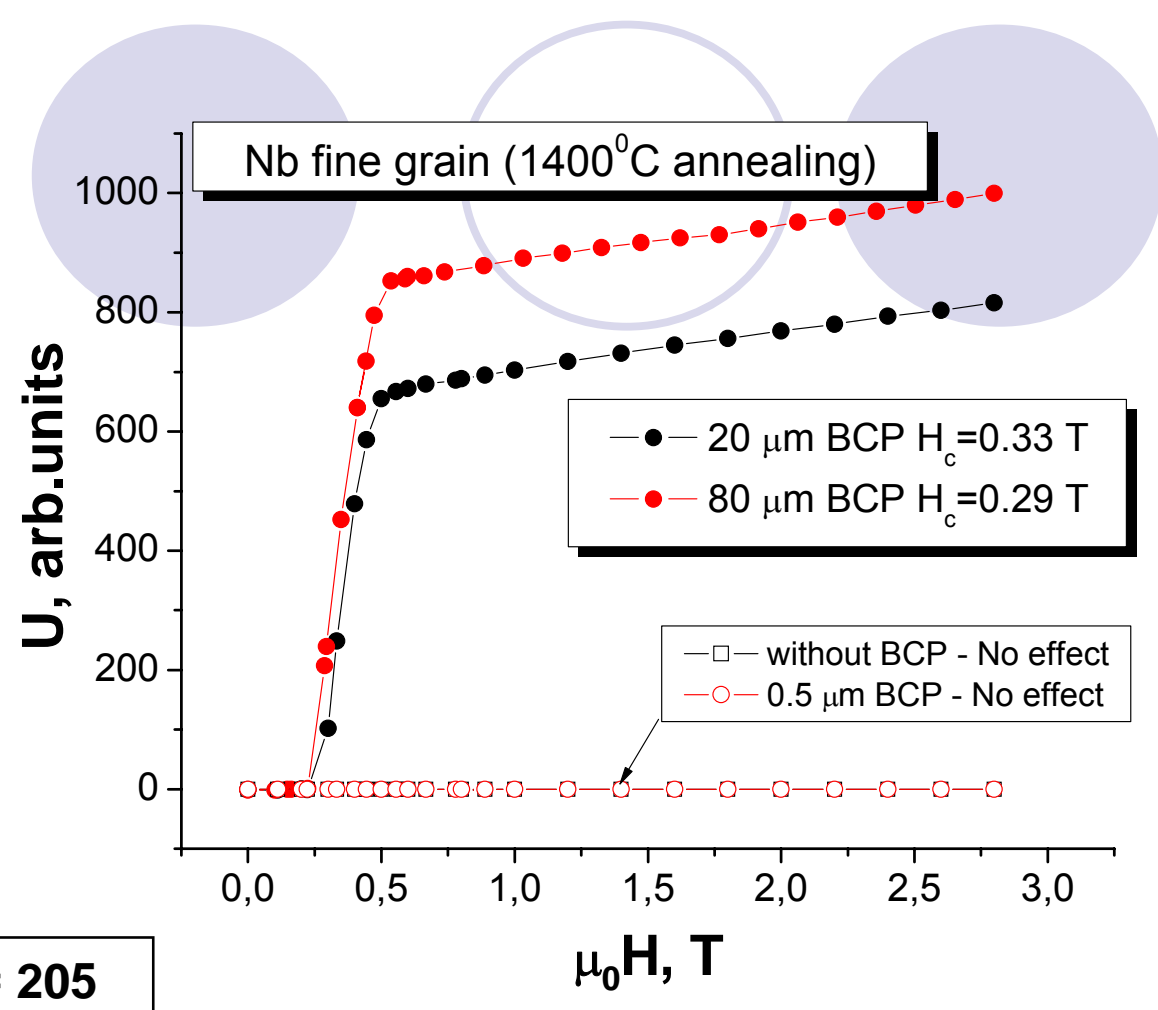

Similar bend at definite magnetic fields was observed on DC magnetic resistance.

This bend is probably caused by transition from SC to normal conducting state of niobium 


\section{Summary}

One more approach for determination the RRR values by means of ACsusceptibility examined

RRR values for main crystallographic orientations of $\mathrm{Nb}$ single crystals are obtained

- Good correlation with results for RRR obtained by 4 point DC method

- The magnetic field dependence of value $R$ follows to the Kapitza law $R=K f(B)$

- The advantage of this method is possibility to measure simultaneously the different magnetic and transport properties such as a very small values of resistivity. Determination of resistivity can be done by taking into account the size and the shape of the sample. 